\title{
O ultrassom no amaciamento de carnes
}

\author{
The ultrasound on meat tenderization
}

\section{Larissa de Lima Alves ${ }^{\text {* }}$ Alexandre José Cichoski ${ }^{I I}$ Juliano Smanioto Barin ${ }^{\text {II }}$ Cristine Rampelotto ${ }^{\mathrm{I}}$ Edgar Cesar Durante ${ }^{\mathrm{II}}$}

\section{RESUMO}

O ultrassom é uma das novas tecnologias limpas aplicadas a alimentos. Na ciência e tecnologia de carnes, é estudado principalmente quanto à sua capacidade de melhorar a maciez da carne, por mecanismos de cavitação. Alguns parâmetros acústicos como frequência, intensidade e tempo de exposição ao tratamento influenciam na tenderização da carne. Os primeiros estudos determinaram que o uso de altas frequências não apresentaram efeitos na textura, em função de não provocarem cavitação. A intensidade de ultrassom que atinge a matriz cárnea também é importante, sendo que, quando aplicada abaixo de $10 \mathrm{~W}$ $\mathrm{cm}^{-2}$ ou muito acima desse valor, não se percebe o efeito. $O$ tempo de exposição é dependente da frequência e intensidade utilizadas e influencia diretamente na maciez. Características de qualidade da carne, como perda de peso após cozimento, queda de $\mathrm{pH}$, cor e microbiologia também foram analisados por diversos autores, com dados contraditórios quanto ao efeito do ultrassom sobre esses parâmetros. As particularidades de cada músculo dificultam as comparações de resultados, abrindo espaço para novas pesquisas. O uso de ultrassom na tecnologia de carnes, visando a melhorar a maciez, mostra-se como uma tecnologia promissora, um potencial a ser explorado.

Palavras-chave: carne, cavitação, maciez, tecnologia limpa.

\section{ABSTRACT}

Ultrasound is one of the new clean technologies applied to food. In science and technology of meat, is mainly studied for its ability to improve meat tenderness by cavitation mechanisms. Some acoustic parameters such as frequency, intensity and exposure time influence the treatment of meat tenderization. Initial studies determined that the use of high frequencies did not show effects on the texture, due to not cause cavitation. The intensity in which the ultrasound reaches the meat matrix is also important, and when applied below $10 \mathrm{~W} \mathrm{~cm}^{-2}$ or much above this value does not realize the effect. The exposure time is dependent on the frequency and the used intensity directly influences the softness. Meat quality characteristics, such as weight loss after cooking, drop in $\mathrm{pH}$, color and microbiology were also analyzed by several authors, with conflicting data on the effect of ultrasound on these parameters. The particularities of each muscle hinder comparisons of results, stimulating new researches. The use of ultrasound technology to improve meat tenderness shows itself as a promising technology with the potential to be exploited.

Key words: cavitation, clean technologies, meat, tenderness.

\section{INTRODUÇÃO}

A tecnologia de alimentos é uma ciência dinâmica em constante evolução, em que se busca concomitantemente atender a expectativa dos consumidores por novos sabores e praticidade, e a necessidade da indústria na redução de custos. As técnicas convencionais de processamento e extração vêm sendo substituídas pelas chamadas tecnologias limpas ou verdes, que tipicamente envolvem menos tempo de processamento, menor gasto de água e energia e menor produção de efluentes e substâncias tóxicas (CHEMAT et al., 2011). Dentre as novas tecnologias, destaca-se o uso de fluidos supercríticos, micro-ondas, campos elétricos pulsados, alta pressão hidrostática, luz ultravioleta, luz ultravioleta pulsante, aquecimento ôhmico e ultrassom (LEADLEY \& WILLIAMS, 2008).

$\mathrm{O}$ ultrassom tem sido explorado desde sua descoberta no começo do século XX para uma

IPrograma de Pós-graduação em Ciência e Tecnologia de Alimentos (PPGCTA), Universidade Federal de Santa Maria (UFSM), Avenida Roraima, 1000, Bairro Camobi, 97105-900, Santa Maria, RS, Brasil. E-mail: larissafarm@ yahoo.com.br. *Autor para correspondência.

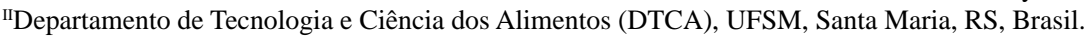


variedade de finalidades na medicina e na indústria química e de engenharia. Com os avanços nos estudos dessa tecnologia e o entendimento de seus mecanismos de ação, surgiu a possibilidade de utilização na área de alimentos. Desde a metade do século passado, o ultrassom vem sendo estudado para finalidades no processamento, preservação e extração de diversas substâncias de alimentos. Diversos estudos têm mostrado, também, sua eficiência nos processos de cozimento, congelamento/descongelamento, cristalização, emulsificação, filtração, moldagem, corte, extração, secagem, desgaseificação, oxidação e maturação em diferentes matrizes alimentares (DOLATOWSKI et al., 2007; LEADLEY \& WILLIAMS, 2008; CHEMAT et al., 2011, CÁRCEL et al., 2012; CHANDRAPALA et al., 2012).

$\mathrm{Na}$ ciência e tecnologia de carnes, as pesquisas com ultrassom estão focadas, principalmente, na capacidade de provocar mudanças nas propriedades físicas e químicas do músculo, interferindo positivamente na maciez da carne (JAYASOORIYA et al., 2004). A maciez é um atributo de importância na avaliação pelo consumidor e diversas técnicas são utilizadas pela indústria para melhorar essa característica. Estudos mostraram que a suspensão da carcaça pelo osso pélvico durante o resfriamento é mais vantajosa para textura da carne, quando comparada ao método tradicional de suspensão pelo tendão de Aquiles; porém, é pouco utilizada em função de demandar mais espaço e dificultar a circulação de ar na câmara fria (EIKELENBOOM et al., 1998; AHNSTRÖM et al, 2012). A estimulação elétrica é usada nas carcaças antes do resfriamento pós-abate para acelerar a glicólise e adiantar o rigor mortis, evitando o encurtamento pelo frio, fenômeno que torna a carne rígida (ORDÓÑEZ, 2005). O uso de enzimas proteolíticas, provindas de plantas, bactérias e fungos, está bem consolidado na literatura; porém, quando usado como solução para imersão da carne, produz excessivo abrandamento superficial, além de odores e sabores desagradáveis. O uso dessas enzimas injetadas no animal vivo poucas horas antes do abate surgiu como alternativa; porém, as enzimas só são ativadas pela temperatura do cozimento, ou seja, no momento da compra, o consumidor não percebe a maciez do produto (PRÄNDL et al., 1994). A injeção de soluções de salmouras, polifosfatos, ácidos e cloretos também auxiliam na melhora da textura, mas afetam a aparência da carne em função do dano causado pelas agulhas (LAWRIE, 2005).

Assim, as técnicas tradicionais de amaciamento possuem algumas desvantagens, abrindo espaço para novas tecnologias. Por ser uma técnica puramente física, sem necessitar de aquecimento ou reações químicas e apresentar bons resultados, o ultrassom surge como uma alternativa interessante. Dessa forma, o uso do ultrassom na ciência e na tecnologia de carnes é discutido, enfatizando os aspectos mais importantes dos parâmetros acústicos do ultrassom que influenciam na maciez e as consequências da sua aplicação sobre a qualidade global da carne.

\section{O ultrassom}

O ultrassom é uma onda mecânica acústica e, como tal, necessita de um meio para se propagar. As ondas acústicas são classificadas tomando a frequência audível pelo ouvido humano, que varia de 20 Hertz $(\mathrm{Hz})$ a 20 kiloHertz $(\mathrm{kHz})$. Frequências abaixo de $20 \mathrm{~Hz}$ são referidas como infrassom e acima de $20 \mathrm{kHz}$ como ultrassom (CÁRCEL et al., 2012). $\mathrm{O}$ ouvido humano não detecta a onda ultrassônica, enquanto alguns animais como os golfinhos e os morcegos utilizam o ultrassom para localização (AWAD et al., 2012).

As ondas ultrassônicas são classificadas em dois grandes grupos, dependendo da sua frequência e intensidade. Ultrassons de baixa energia são de alta frequência (2-20 Megahertz - MHz) e baixa intensidade $\left(<1 \mathrm{~W} \mathrm{~cm}^{-2}\right)$ e não são destrutivos, tendo emprego em técnicas de imagens não invasivas, sensores e análises na medição da composição, maturação, eficácia de emulsificação e concentração ou dispersão de partículas em fluidos (LEADLEY \& WILLIAMS, 2008). Na ciência e tecnologia de alimentos, são utilizados em técnicas analíticas para prover informações sobre propriedades físico-químicas, composição, estrutura e estado físico de alimentos. $\mathrm{O}$ uso do ultrassom nesses casos apresenta vantagens de ser uma técnica rápida, não invasiva, não destrutiva e precisa (DOLATOWSKI et al., 2007).

Ondas ultrassônicas de alta energia são de baixas frequências (20-100 kHz) e desenvolvem níveis de potência mais altos $\left(10-1000 \mathrm{~W} \mathrm{~cm}^{-2}\right)$, com energia suficiente para romper enlaces intermoleculares, sendo que intensidades superiores a $10 \mathrm{~W} \mathrm{~cm}$ originam efeitos de cavitação, capazes de modificar algumas propriedades físicas e favorecer reações químicas (PIYASENA et al., 2003; JAYASOORIYA et al., 2004).

O fenômeno de cavitação, responsável por boa parte dos efeitos do ultrassom sobre a matriz alimentar, origina-se quando a onda ultrassônica atravessa um meio líquido, provocando alternância de ondas de compressão (pressão positiva) e rarefação (pressão negativa), produzindo bolhas no 
líquido. Durante o ciclo de expansão, ocorre a difusão de gases para o interior da bolha, promovendo sua expansão. No caso da cavitação estável, a oscilação compressão/rarefação é regular e as bolhas induzem microagitação no líquido sem implodir. Na cavitação instável ou transiente, essas bolhas oscilam entre os estados de compressão/rarefação até ocorrer a sua implosão, gerando um aumento de pressão e temperatura localizado e a produção de microjatos que apresentam energia suficiente para desintegrar células e desnaturar enzimas (PIYASENA et al., 2003; CHEMAT et al., 2011, CÁRCEL et al., 2012). O efeito de cavitação é importante no estudo da inativação de microrganismos por provocar afinamento da membrana citoplasmática, aquecimento localizado e produção de radicais livres nocivos aos microrganismos (PIYASENA et al., 2003). Contudo, são necessárias intensidades muito altas de ultrassom para efeito esterilizante efetivo, sendo acoplado a outras técnicas descontaminantes como pressão (manosonicação), calor (termosonicação) ou ambos (manotermosonicação) em estudos recentes (DOLATOWSKI et al., 2007).

O ultrassom é produzido a partir de um transdutor, que converte a energia elétrica em energia mecânica sonora com frequências ultrassônicas. Esses transdutores geralmente são feitos de materiais cerâmicos piezoelétricos, como o titanato zirconato de chumbo (PZT), o titanato de bário e o metaniobato de chumbo. Os sistemas de aplicação de ultrassom mais usados na tecnologia de alimentos são os banhos e as sondas. Os banhos possuem o transdutor unido à base ou às paredes do tanque e a energia ultrassônica é cedida diretamente ao líquido que preenche o recipiente, geralmente a água, que transfere essa energia ao alimento imerso nesse líquido. No sistema de sondas, o sinal acústico é amplificado e dirigido diretamente ao alimento através da haste metálica, sem necessidade de adição de um meio líquido entre alimento e sonda (LEADLEY \& WILLIAMS, 2008).

\section{Ultrassom e o amaciamento de carnes}

O ultrassom de alta frequência tem seu uso na ciência e tecnologia de carnes baseado na variação de sua velocidade a diferentes temperaturas, em porções magras e gordurosas, podendo ser usado para medir a quantidade de gordura, umidade e proteína de animais vivos e de cortes. Desde a década de 50, o ultrassom é utilizado para inferir o conteúdo de gordura em bovinos vivos. Atualmente, utiliza-se também para identificar datas para abate e predizer a qualidade e palatabilidade das carcaças bovinas, suínas e ovinas, além de ser utilizado em técnicas de análise para determinar a composição físico-química de peixes e frangos (DOLATOWSKI et al., 2007; AWAD et al., 2012).

Contudo, a maioria dos estudos na área de carnes utiliza o ultrassom de baixa frequência, estudando seu efeito na textura. A maciez é um dos principais parâmetros de qualidade da carne e um dos principais problemas da indústria cárnea. A impressão geral da maciez para o paladar inclui a textura e envolve três aspectos. Primeiramente, a facilidade de penetração da carne pelos dentes; em segundo lugar, a facilidade com a qual a carne se fragmenta; e, em terceiro lugar, a quantidade de resíduo que permanece após a mastigação. Inúmeros fatores pré e pós-abate influenciam na maciez, como a espécie, raça, idade, músculo, quantidade de gordura intra e intermuscular, velocidade de queda de $\mathrm{pH}$, exposição a baixas temperaturas (encurtamento pelo frio), condições de cocção, entre outros (LAWRIE, 2005). A maciez é determinada por dois principais componentes do músculo esquelético: o tecido contrátil ou fração miofibrilar (actina, miosina, tropomiosina) e o tecido conjuntivo (colágeno, elastina, reticulina), sendo o primeiro facilmente manipulado pelas técnicas artificiais de amaciamento pós-abate. O tecido conjuntivo é mais difícil de manipular para aumentar a maciez (JAYASOORIYA et al., 2004).

Em condições naturais, a maciez é obtida pela maturação, que ocorre após o rigor mortis. Os processos metabólicos da conversão do músculo em carne encerram-se após a conclusão do rigor mortis e a carne pronta para o consumo é obtida após certo tempo de armazenamento refrigerado, que pode durar desde dias até semanas. Durante a maturação, enzimas proteolíticas, principalmente as catepsinas e calpaínas, atuam degradando actina, miosina e proteínas da faixa Z. As catepsinas são capazes de hidrolisar as proteínas miofibrilares, inclusive os segmentos peptídicos que resultam dessa hidrólise. Entretanto, as calpaínas são as enzimas que exercem maior efeito sobre o amaciamento, requerendo para sua ativação doses de cálcio na ordem de micromoles (calpaína I) ou milimoles (calpaína II), sendo a primeira a responsável pela maior parte do efeito na textura da carne (PRÄNDL et al., 1994; ORDÓÑEZ, 2005).

$\mathrm{O}$ efeito do ultrassom sobre a textura da carne é basicamente devido aos efeitos de cavitação, principalmente cavitação transiente (CHANDRAPALA et al., 2012). A natureza destrutiva da cavitação e a vibração da onda ultrassônica levam ao enfraquecimento da estrutura muscular, com a ruptura das estruturas do colágeno e das miofibrilas (GOT et al., 1999; STADNIK \& DOLATOWSKI, 
2011). Além disso, a cavitação também causa danos à estrutura de organelas celulares, com a liberação de catepsinas dos lisossomos e de cálcio do retículo sarcoplasmático, o qual ativará o sistema calpaína, acelerando a proteólise (CHANDRAPALA et al., 2012). A atividade enzimática de proteases também poderá ser acelerada pelo aumento de temperatura que ocorre durante a exposição ao ultrassom (DICKENS et al., 1991; CHANDRAPALA et al., 2012). Outra consequência desse aquecimento é a desnaturação proteica, que poderia vir a contribuir com o amaciamento do músculo (JAYASOORIYA et al., 2007).

Para obter efeitos sobre a textura, parâmetros acústicos como frequência, intensidade, duração da exposição e temperatura devem ser considerados, além das características do músculo analisado. Desde os primeiros estudos, busca-se inferir o efeito isolado desses fatores e qual a melhor combinação para o efeito desejado.

A frequência de ultrassom é um dos aspectos mais importantes para o tratamento de carnes. GOT et al. (1999) utilizaram alta frequência (2,6 $\mathrm{MHz}$ ) com intensidade de $10 \mathrm{~W} \mathrm{~cm}^{-2}$ por 30 segundos para amaciar músculos bovinos na fase de pré e pós rigor mortis, sem obter melhora nos parâmetros de maciez considerados. Frequências na ordem de $\mathrm{MHz}$ não são capazes de provocar efeitos de cavitação, o que justificou o resultado negativo naquele estudo (PIYASENA et al., 2003). A cavitação ocorre entre 20 e $100 \mathrm{kHz}$, sendo essa faixa de frequência utilizada para estudos posteriores, que mostraram efetividade na maciez, especialmente entre 20 e 45 kHz (JAYASOORIYA et al., 2007; STADNIK et al., 2008; STADNIK \& DOLATOWSKI, 2011).

A intensidade com que o ultrassom atinge o músculo também deve ser considerada. O trabalho de ZAYATAS et al. (1971) é um dos primeiros estudos sobre o uso de ultrassom em matriz cárnea com a finalidade de melhorar a maciez, utilizando baixa intensidade $\left(2 \mathrm{~W} \mathrm{~cm}^{-2}\right)$ em frequência de $20 \mathrm{kHz}$. Os autores não verificaram nenhum efeito significativo, sugerindo que intensidades maiores são necessárias. Estudos seguintes que avaliaram baixas intensidades $\left(<10 \mathrm{~W} \mathrm{~cm} \mathrm{~cm}^{-2}\right)$ também não observaram resultados efetivos. POHLMAN et al. (1997a), ao testarem frequência de $20 \mathrm{kHz}$ e $1,55 \mathrm{~W} \mathrm{~cm}^{-2}$ de intensidade por 8,16 ou 24 minutos de exposição à sonda de ultrassom, não observaram efeito sobre a textura nos músculos Semitendinosus e Biceps femoris em bovinos, avaliada através da medida da força de cisalhamento. Em um segundo estudo, POHLMAN et al. (1997b) utilizaram a mesma frequência $(20 \mathrm{kHz})$, porém com maior intensidade $\left(22 \mathrm{~W} \mathrm{~cm}^{-2}\right)$ por 0,5 ou 10 minutos em músculo bovino Pectoralis, sem melhorar a maciez e as características sensoriais e de cozimento. $\mathrm{O}$ efeito observado foi atribuído à grande quantidade de colágeno do músculo analisado, que pode ter mascarado o efeito do ultrassom. LYNG et al. (1997) estudaram aplicação de ultrassom com baixas intensidades (30-47 kHz e 0,29-0,62 $\mathrm{W} \mathrm{cm}^{-2}$ de frequência e intensidades, respectivamente) em banho sobre os músculos Longissimus, Semitendinosus e Biceps femoris, sem obter resultado efetivo sobre amaciamento, solubilidade de colágeno e proteólise miofibrilar. Assim como baixas intensidades não apresentam resultados positivos, a exposição a intensidades maiores $\left(62 \mathrm{~W} \mathrm{~cm}^{-2}\right.$ com $20 \mathrm{kHz}$ de frequência) não foi eficaz, quando aplicada por 30 segundos em músculos bovinos e de cordeiro (LYNG et al., 1998 a,b). Os autores atribuíram o resultado à alta intensidade e ao baixo tempo de exposição utilizados.

O tempo de exposição ao ultrassom também exerce efeito importante quando se busca amaciamento da carne. JAYASOORIYA et al. (2007) avaliaram o efeito do tempo de exposição ( 0 , $30,60,120$ e 240 segundos) de músculos bovinos Semitendinosus e Longissimus a uma sonda com frequência de $24 \mathrm{kHz}$ e $12 \mathrm{~W} \mathrm{~cm}^{-2}$ de intensidade, analisando diversos parâmetros como $\mathrm{pH}$, cor, mudanças nas perdas de peso após exposição ao ultrassom e após cozimento, além de avaliar as mudanças de textura provocadas pela sonicação, concluindo que são necessários tempos de exposição ao ultrassom acima de 60 segundos para se ter efeito na tenderização dos músculos. Os autores também relataram que, à medida que o tempo de exposição aumentou, a firmeza da carne reduziu, sem prejudicar os demais parâmetros de qualidade avaliados.

STADNIK et al. (2008) utilizaram banho de ultrassom para acelerar o processo de maturação de músculo bovino Semimembranosus à frequência de $45 \mathrm{kHz}$, na intensidade de $2 \mathrm{~W} \mathrm{~cm}^{-2} \mathrm{e}$ exposição por 120 segundos. As amostras sonicadas apresentaram mudanças post mortem mais avançadas que o controle não sonicado, com maior capacidade de retenção de água no músculo e maior degradação de proteínas, sendo que os músculos expostos ao ultrassom ficaram mais macios do que os músculos do controle. Em trabalho posterior, foram usados os mesmos parâmetros acústicos $\left(45 \mathrm{kHz}, 2 \mathrm{~W} \mathrm{~cm}{ }^{-2}\right.$, 120 s) e foi constatado que, 48 e 72 horas após o abate, o valor da força de cisalhamento usado para estimar a maciez foi significativamente menor na amostra exposta ao ultrassom. Após 96 horas, os valores obtidos para amostra sonicada e controle 
foram similares, ou seja, o ultrassom exerceu efeito sobre a maciez apenas nos três primeiros dias após o abate (STADNIK \& DOLATOWSKI, 2011). No trabalho de JAYASOORIYA et al. (2007), os autores observaram efeito do ultrassom até o quinto dia post-mortem, sendo que, após 8,5 dias, o efeito foi semelhante ao controle não sonicado. Sendo assim, sugere-se que o ultrassom apenas adianta o processo de maturação natural, reduzindo o tempo necessário para que a carne fique macia.

Além da maciez, outros parâmetros importantes para a qualidade da carne devem ser ponderados quando o músculo é exposto ao ultrassom. Os valores da queda do $\mathrm{pH}$ post-mortem foram avaliados por alguns autores e os resultados ainda são contraditórios. STADNIK \& DOLATOWSKI (2011) não observaram efeito significativo do ultrassom sobre esse parâmetro. JAYASOORIYA et al. (2007) relatam aumento de $\mathrm{pH}$ conforme o tempo de exposição ao ultrassom variou de 0 a 240 segundos, atribuindo isso ao fato da cavitação causar danos à célula com liberação de íons no citosol e mudanças na conformação de proteínas que ocultariam grupos acídicos (GOT et al., 1999). A queda de pH é importante em função de estar diretamente ligada à ativação das enzimas proteolíticas calpaínas e catepsinas. $\mathrm{O}$ pH ótimo para ação das catepsinas é moderadamente ácido, enquanto das calpaínas é neutro. Desse modo, o aumento de $\mathrm{pH}$ aumenta a atividade das calpaínas, as quais têm papel principal na maturação. STADNIK et al. (2008) observaram efeito significativo do ultrassom sobre o $\mathrm{pH}$ apenas após $48 \mathrm{~h}$ post-mortem, quando a amostra sonicada apresentou valor de $\mathrm{pH}$ significativamente mais alto que o controle, mas semelhante nos tempos de análise de 24, 72 ou 96 h post-mortem.

As perdas de peso após exposição ao ultrassom e ao cozimento foram medidas por POHLMAN et al. (1997a,b) e por JAYASOORIYA et al. (2007) e nenhum efeito do ultrassom foi observado, quando comparado ao controle não sonicado. STADNIK et al. (2008) avaliaram a capacidade de retenção de água em amostras controle e sonicadas em banho a $45 \mathrm{kHz}, 2 \mathrm{~W} \mathrm{~cm}^{-2}$ por 120 segundos, e não observaram diferença após 24 ou 48 horas postmortem. Esse é um aspecto positivo, uma vez que o conteúdo de água contribui para as características de suculência da carne.

A cor também é um atributo bastante considerado pelo consumidor no momento da compra. Os parâmetros de cor $\mathrm{L}^{*}$ (luminosidade), $a^{*}$ (verde-vermelho) e b* (amarelo-azul) não foram afetados pelo tratamento de ultrasssom nos estudos de JAYASOORIYA et al. (2007) e STADNIK \&
DOLATOWSKI et al. (2011). CHANG et al. (2012) não observaram efeito do ultrassom sobre $\mathrm{L}^{*} \mathrm{e} \mathrm{a}^{*}$, mas o parâmetro $b^{*}$ foi significativamente menor que o controle, quando exposto por 30 segundos . Esse efeito foi atribuído à alta frequência utilizada (40 $\mathrm{kHz}$ ), especialmente quando comparada à frequência testada por JAYASOORIYA et al. (2007) (24 kHz). No primeiro estudo de POHLMAN et al. (1997a), ao usar banho de ultrassom com parâmetros acústicos de $20 \mathrm{kHz}, 1,55 \mathrm{~W} \mathrm{~cm}^{-2}$ e exposição por $8,16 \mathrm{ou}$ 24 minutos em músculo bovino Semitendinosus e Biceps femoris, não foi percebido efeito do ultrassom sobre os parâmetros $\mathrm{L}^{*}, \mathrm{a}^{*} \mathrm{ou} \mathrm{b}^{*}$. Porém, ao manter a frequência de $20 \mathrm{kHz}$ e aumentar a intensidade para $22 \mathrm{~W} \mathrm{~cm}^{-2}$ com tempos de 0,5 ou 10 minutos em músculo Pectoralis, os autores relatam maior luminosidade (maior $\mathrm{L}^{*}$ ), menor intensidade da coloração vermelha (menor $\mathrm{a}^{*}$ ) e maior intensidade de tonalidade amarela (maior $\mathrm{b}^{*}$ ), quando o músculo foi exposto ao ultrassom, embora não se tenha percebido diferença entre os tempos de 5 e de 10 minutos, ou seja, o ultrassom afetou os parâmetros de cor, independente do tempo de exposição utilizado (POHLMAN et al., 1997b).

$\mathrm{O}$ efeito do ultrassom sobre a flora benéfica, deteriorante e patogênica em carnes ainda é pouco explorado, embora já se saiba que o ultrassom não é efetivo quando utilizado como único tratamento para reduzir a carga microbiana. POHLMAN et al. (1997a) avaliaram o potencial germicida do ultrassom em músculos bovinos, sem observar diferença estatisticamente significante quando as amostras sonicadas foram comparadas ao controle por 30 dias de armazenamento. Apenas no dia zero foi verificado leve efeito inibitório do ultrassom sobre o número de colônias, mas a diferença entre a amostra sonicada e o controle desapareceu após cinco dias. Desse modo, o ultrassom exerceu apenas impacto imediato sobre o crescimento de microrganismos, sem garantir o efeito ao longo do armazenamento.

Contudo, é difícil comparar dados, em função da variação das características específicas de equipamentos e das peculiaridades intrínsecas de cada músculo estudado. Diversos fatores podem influenciar nos resultados, desde as condições acústicas até as particularidades de cada músculo, já que alguns efeitos do uso do ultrassom na qualidade da carne ainda são contraditórios.

\section{CONCLUSÃO}

O ultrassom é uma tecnologia de processamento nova na área de alimentos e com 
potencial de ser utilizado na ciência e tecnologia de carnes para auxiliar na obtenção de carnes mais macias, tão desejadas pelo consumidor. Alguns parâmetros acústicos que influenciam na sua efetividade, bem como mecanismos de ação, já foram esclarecidos; contudo, alguns efeitos na qualidade da carne ainda são contraditórios. Sendo assim, o uso do ultrassom na área de carnes carece de mais estudos e deve ser melhor explorado, abrindo espaço para desenvolvimento de novas tecnologias acessórias e o estabelecimento de parâmetros que possam padronizar sua aplicação e tornar seu efeito reprodutível.

\section{REFERÊNCIAS}

AHNSTRÖM, M.L. et al. Effects of pelvic suspension of beef carcasses on quality and physical traits of five muscles from four gender-age groups. Meat Science, v.90, n.3, p.528-535, 2012. Disponível em: <http://www.sciencedirect. com/science? ob=ArticleListURL\& method=list\& ArticleListID $=2138146154 \&$ _sort $=r \&$ _st $=13 \&$ view $=c \&$ _ acct $=$ C000067446\&\&_version $=1 \& \_u r l$ Version $=0$ \&_userid $=54772$ $35 \& m d 5=b 491 \mathrm{f} 6002 \mathrm{f} 93 \mathrm{cc} 5 \mathrm{a} 9 \mathrm{ef} 3 \mathrm{a} 89 \mathrm{e} 14 \mathrm{fec} 58 \mathrm{f} \&$ searchtype $=\mathrm{a}>$. Acesso em: 12 maio 2012. doi: 10.1016/j.meatsci.2011.09.003.

AWAD, T.S. et al. Applications of ultrasound in analysis, processing and quality control of food: a review. Food Research International, v.48, n.2, p.410-427, 2012. Disponível em: <http://www.sciencedirect.com/science?_ob=ArticleListURL\&_ method=list\&_ArticleListID $=2137572846 \&$ \& sort $=r \&$ _ $\mathrm{st}=13 \& \mathrm{view}=\mathrm{c} \&$ _acct $=$ C $000067446 \&$ _version $=1 \&$ _ urlVersion $=0 \& \_$userid $=5477235 \& \mathrm{md} 5=\mathrm{e} 5428 \mathrm{dc} 2 \mathrm{c} 5554 \mathrm{a} 5 \mathrm{a} 20 \mathrm{c} 0$ 4d2e764f3e89\&searchtype=a.>. Acesso em: 14 jun. 2012. doi: 10.1016/j.foodres.2012.05.004

CÁRCEL, J.A. et al. Food process innovation through new technologies: use of ultrasound. Journal of Food Engineering, v.110, n.2, p.200-207, 2012. Disponível em: <http:// www.sciencedirect.com/science?_ob=ArticleListURL\&_ method=list\&_ArticleListID $=2137575294 \&$ sort $=$ r\& $\mathrm{st}=13 \& \mathrm{view}=\mathrm{c} \&$ _acct $=$ C $000067446 \&$ _version $=1 \&$ _ urlVersion $=0 \&$ _userid $=5477235 \& \mathrm{md} 5=3432 \mathrm{e} 4 \mathrm{~d} 13 \mathrm{cc} 582 \mathrm{e} 9662$ 81a89d8411fca\&searchtype $=\mathrm{a}>$. Acesso em: 25 maio 2012. doi 10.1016/j.jfoodeng.2011.05.038.

CHANDRAPALA, J. et al. Ultrasonics in food processing. Ultrasonics Sonochemistry, v.19, n.5, p.975983, 2012. Disponível em: <http://www.sciencedirect. com/science?_ob=ArticleListURL\&_method=list\&_ ArticleListID $=2137584131 \&$ \&sort $=r \& \_s t=13 \&$ view $=c \&$ _ acct $=$ C000067446\& version $=1 \&$ urlVersion $=0 \&$ userid $=54772$ $35 \&$ md5=e2cb57fa4934d31f3653687a20951bc6\&searchtype=a . Acesso em: 12 jun. 2012. doi: 10.1016/j.ultsonch.2012.01.010.

CHANG, H.J. et al. Effects of characteristics changes of collagen on meat physicochemical properties of beef semitendinosus muscle during ultrasonics processing. Food and Bioprocess Technology, v.5, n.1, p.285-297, 2012. Disponível em: <http:// www.springerlink.com/content/277055m3766q0641/>. Acesso em: 20 set. 2012. doi: 10.1007/s11947-009-0269-9.

CHEMAT, F. et al. Applications of ultrasound in food technology: processing, preservation and extraction. Ultrasonics
Sonochemistry, v.18, n.4, p.813-835, 2011. Disponível em: <http: //www.sciencedirect.com/science?_ob=ArticleListURL\&_ method=list\&_ArticleListID $=2137594603 \&$ \&sort $=r \&$ _ $\mathrm{st}=13 \& \mathrm{view}=\mathrm{c} \&$ acct $=\mathrm{C} 000067446 \&$ _version $=1 \&$ urlVersion $=0 \&$ userid $=5477235 \& \mathrm{md} 5=9 \mathrm{df} 27 \mathrm{fc} 288770736242$ 87ff72065988d\&searchtype $=\mathrm{a}>$. Acesso em: 16 abr. 2012. doi: 10.1016/j.ultsonch.2010.11.023.

DICKENS, J.A. et al. Effect of ultrasonic irradiation on some physical characteristics of broiler breast muscle and cooked meat. Poultry Science, v.70, n.1, p.389-396, 1991. Disponível em: $<$ http://ps.fass.org/search?submit=yes\&pubdate_year=\&volume= $70 \&$ firstpage $=389 \&$ doi $=\&$ author $1=\&$ author $2=\&$ title $=\&$ andorexac ttitle $=$ and $\&$ titleabstract $=\&$ andorexacttitleabs $=$ and $\&$ fulltext $=\&$ and orexactfulltext=and $\&$ fmonth $=\&$ fyear $=\&$ tmonth $=\&$ tyear $=\&$ format $=$ standard\&hits $=10 \&$ sortspec $=$ relevance $\&$ submit $=$ yes \&submit $=S$ ubmit>. Acesso em: 10 out. 2012. doi:10.3382/ps.0700389.

DOLATOWSKI, Z.J. et al. Applications of ultrasound in food technology. Technologia Alimentaria, v.6, n.3, p.89-99, 2007. Disponível em: 〈http://www.food.actapol.net/pub/8_3_2007.pdf>. Acesso em: 10 set. 2012.

EIKELENBOOM, G. et al. Effect of pelvic suspension and cooking temperature on the tenderness of electrically stimulated and aged beef, assessed with shear and compressed tests. Meat Science, v.49, n.1, p.89-99, 1998. Disponível em: <http://www.sciencedirect. $\mathrm{com} / \mathrm{science} ? \mathrm{ob}=$ ArticleListURL\& method=list\& ArticleListID $=2137600892 \&$ \&sort $=r \&$ \& $=13 \&$ view $=c \&$ _ acct $=$ C000067446\&_version $=1$ \&_urlVersion $=0$ \&_userid $=54772$ $35 \& \mathrm{md} 5=0 \mathrm{da} 74 \mathrm{e} 409 \mathrm{f} 5 \mathrm{a} 852 \mathrm{f047bf} 1 \mathrm{c} 183719960 \&$ searchtype $=\mathrm{a}>$. Acesso em: 12 jun. 2012.

GOT, F. et al. Effects of high-intensity high frequency ultrasound on ageing rate, ultrastructure and some physic-chemical properties of beef. Meat Science, v.51, n.1, p.35-42, 1999. Disponível em: <http://www.sciencedirect.com/science?_ob=ArticleListURL\&_ method=1ist\&_ArticleListID $=2137606253 \&$ \&sort $=r \&$ _ $\mathrm{st}=13 \& \mathrm{view}=\mathrm{c} \&$ acct $=\mathrm{C} 000067446 \&$ \&ersion $=1 \&$ urlVersion $=0 \& \_u s e r i d=5477235 \& \mathrm{md} 5=0319 \mathrm{~b} 36127 \mathrm{ae} 877484 \mathrm{ac} 5$ edebd54ed2f\&searchtype=a $>$. Acesso em: 15 set. 2012.

JAYASOORIYA, S.D. et al. Effect of high power ultrasound waves on properties of meat: a review. International Journal of Food Properties, v.7, n.2, p.301-319, 2004.

JAYASOORYA, S.D. et al. Effect of high power ultrasound and ageing on the physical properties of bovine Semitendinosus and Longissimus muscles. Meat Science, v.75, p.628-639, 2007. Disponível em: <http://www.sciencedirect.com/science?_ob=ArticleListURL\&_ method=1ist\&_ArticleListID $=2137609209 \&$ _sort $=r \&$ _ $\mathrm{st}=13 \& \mathrm{view}=\mathrm{c} \& \_$acct $=\mathrm{C} 000067446 \& \_$version $=1 \&$ urlVersion $=0 \& \_u s e r i d=5477235 \& \mathrm{md} 5=\mathrm{b} 567$ ada $58 \mathrm{c} 32 \mathrm{bba} 0401$ 3a4677b6d0d59\&searchtype $=a>$. Acesso em 22 jul. 2012. doi: 10.1016/j.meatsci.2006.09.010.

LAWRIE, R.A. Ciência da carne. 6.ed. Porto Alegre: Artmed, 2005. 384p.

LEADLEY, E.; WILLIAMS, A. Procesado com pulsos electricos, ultrasonidos de potencia y otras tecnologias emergentes. In: BRENNAM, J.G. Manual del procesado de los alimentos. Zaragoza: Acribia, 2008. Cap.7, p.203-237.

LYNG, J.G. et al. The influence of high intensity ultrasound bath on aspects of beef tenderness. Journal of Muscle Foods, v.8, n.3, 
p.237-249, 1997. Disponível em: <http://onlinelibrary.wiley.com/ doi/10.1111/j.1745-4573.1997.tb00630.x/abstract>. Acesso em: 21 set. 2012. doi: 10.1111/j.1745-4573.1997.tb00630.x.

LYNG, J.G. et al. The effect on aspects of beef tenderness of pre and post rigor exposure to a high intensity ultrasound probe. Journal Science of Food and Agriculture, v.78, n.3, p.308-314, 1998a. Disponível em: <http://onlinelibrary.wiley. com/doi/10.1002/(SICI)1097-0010(199811)78:3\%3C308::AIDJSFA123\%3E3.0.CO;2-F/abstract>. Acesso em: 10 out. 2012. doi: 10.1002/(SICI)1097-0010(199811)78:3<308::AIDJSFA123>3.0.CO;2-F.

LYNG, J.G. et al. The effects of pre and post rigor high intensity ultrasound treatment on aspects of lamb tenderness. LebensmittelWissenschaft und-Technologie, v.31, n.4, p.334-338, 1998 b. Disponível em: <http://www.sciencedirect.com/science/article/ pii/S0023643897903613>. Acesso em: 01 out. 2012. doi: 00236438/98/040334.

ORDÓÑEZ, J.A. Tecnologia de alimentos: alimentos de origem animal. Porto Alegre: Artmed, 2005. 280p.

PIYASENA, P. et al. Inactivation of microbes using ultrasound: a review. International Journal of Food Microbiology, v.87, p.207-216, 2003. Disponível em: <http://www.sciencedirect. com/science?_ob=ArticleListURL\&_method=list\&_ ArticleListID $=2137634184 \&$ \&sort $=r \&$ \&t $=13 \& \mathrm{view}=\mathrm{c} \&$ _ acct $=$ C000067446\&_version $=1 \&$ \&urlVersion $=0 \&$ \&userid $=\overline{5}$ $477235 \&$ md5 $=$ c9768165be503b70cbaaab4cc 185 b49b\&sear chtype=a>. Acesso em: 27 set. 2012. doi: 10.1016/S01681605(03)00075-8.

POHLMAN, F.W. et al. The effect of low-intensity ultrasound treatment on shear properties, color stability and shelf-life of vacuumpackaged beef semitendinosus and biceps femoris muscles. Meat Science, v.45, n.3, p.329-337, 1997a. Disponível em: <http://www. sciencedirect.com/science?_ob=ArticleListURL\&_method=list\&_
ArticleListID $=2137635300 \&$ \&sort $=r \&$ _st $=13 \& \mathrm{view}=\mathrm{c} \&$ _ acct $=$ C000067446\&_version $=1 \&$ \&urlVersion $=0 \&$ _userid $=54772$ $35 \& \mathrm{md} 5=\mathrm{d} 1 \mathrm{f} 4 \mathrm{~b} 29 \mathrm{a} 693356 \mathrm{fee} 3 \mathrm{a} 57455 \mathrm{fce} 083 \mathrm{ba} \&$ searchtype $=\mathrm{a}>$. Acesso em: 24 set. 2012.

POHLMAN, F.W. et al. Effects of high intensity ultrasound treatment, storage time and cooking method on shear, sensory, instrumental color and cooking properties of packaged and unpackaged beef pectoralis muscle. Meat Science, v.46, n.1, p.89-100, 1997b. Disponível em: <http://www.sciencedirect. com/science?_ob=ArticleListURL\&_method=list\&_ ArticleListID $=2137638428 \&$ \&sort $=r \&$ _st $=13 \&$ view $=c \&$ _ acct $=$ C000067446\&_version $=1 \&$ \&urlVersion $=0 \&$ _userid $=5477 \overline{2}$ $35 \& m d 5=9 \mathrm{eb} 85 \mathrm{e} 1 \mathrm{bd} 7 \mathrm{af0bd} 7 \mathrm{f} 4 \mathrm{dbfcdf} 8 \mathrm{a} 5 \mathrm{e} 263 \mathrm{~d} \&$ searchtype $=a>$. Acesso em: 15 set. 2012.

PRÄNDL, O. et al. Tecnología e higiene de la carne. Zaragoza: Acribia, 1994. 853p.

STADNIK, J. et al. Effect of ultrasound treatment on water holding properties and microstructure of beef ( $M$. semimembranosus) during ageing. LWT - Food Science and Technology, v.41, n.10, p.2151-2158, 2008. Disponível em: <http://www.sciencedirect. com/science?_ob=ArticleListURL\&_method=1ist\&_ ArticleListID $=2137638974 \&$ _sort $=r \&$ _st $=13 \&$ view $=c \&$ _ acct $=$ C000067446\&_version $=1 \&$ _urlVersion $=0 \&$ _userid $=54772 \overline{3}$ $5 \&$ md5=38a567c845654f21d1049cb352e1b8f5\&searchtype=a $>$. Acesso em: 22 set. 2012. doi: 10.1016/j.1wt.2007.12.003.

STADNIK, J.; DOLATOWSKI, Z.J. Influence of sonication on Warner-Bratzler shear force, colour and myoglobin of beef (M.semimembranosus). European Food Research Technology, v.233, p.553-559, 2011. Disponível em: <http://link.springer.com/ article/10.1007/s00217-011-1550-5>. Acesso em: 20 set. 2012. doi: 10.1007/s00217-011-1550-5.

ZAYATAS, Y. Effect of ultrasound on animal tissues. MyasnayaIndustriya SSSR, v.42, n.3, p.33-35, 1971. 